\title{
Article \\ Outcomes of Acute Coronary Syndrome in Hospitalized Patients with Celiac Disease, a United States Nationwide Experience
}

\author{
Kamesh Gupta ${ }^{1}$, Khalid Sawalha ${ }^{1}$, Shayal Pundlik ${ }^{1}$, Mohammad Abozenah ${ }^{2}$, Khadija Naseem ${ }^{3}$, \\ Mohammad Al-Akchar ${ }^{4, *}$ and Ahmad Khan ${ }^{5}$ \\ 1 Department of Internal Medicine, UMMS-Baystate, Springfield, MA 01199, USA; \\ Kameshg9@gmail.com (K.G.); khalid.sawalhamd@baystatehealth.org (K.S.); \\ shayal.pundlik@baystatehealth.org (S.P.) \\ 2 Department of Cardiovascular Medicine, UMMS-Baystate, Springfield, MA 01199, USA; \\ mohammed.abozenahmd@baystatehealth.org \\ 3 Department of Medicine, West Virginia University, Charleston, WV 26506, USA; khadija.naseem@camc.org \\ 4 Department of Cardiovascular Medicine, Southern Illinois University, Springfield, IL 62901, USA \\ 5 Division of Gastroenterology, Department of Internal Medicine, Case Western University, \\ Cleveland, OH 44106, USA; ahmad.khan@uhhospitals.org \\ * Correspondence: mal-alakchar68@siumed.edu
}

check for updates

Citation: Gupta, K.; Sawalha, K.; Pundlik, S.; Abozenah, M.; Naseem, K.; Al-Akchar, M.; Khan, A. Outcomes of Acute Coronary Syndrome in Hospitalized Patients with Celiac Disease, a United States Nationwide Experience. Hearts 2022, 3, 38-44. https://doi.org/10.3390/ hearts3010006

Academic Editor: Matthias Thielmann

Received: 23 January 2022 Accepted: 20 February 2022 Published: 1 March 2022

Publisher's Note: MDPI stays neutral with regard to jurisdictional claims in published maps and institutional affiliations.

Copyright: (C) 2022 by the authors. Licensee MDPI, Basel, Switzerland. This article is an open access article distributed under the terms and conditions of the Creative Commons Attribution (CC BY) license (https:// creativecommons.org/licenses/by/ $4.0 /)$.

\begin{abstract}
Background: Cardiovascular disease remains the leading cause of death in the United States. Coronary artery disease alone accounted for approximately 13\% of deaths in the US in 2016. Some studies have suggested an increased prevalence of coronary artery disease (CAD) in chronic inflammatory conditions, such as celiac disease (CD). Chronic subclinical systemic inflammation, decreased absorption of cardio-protective nutrients and drugs have all been postulated as the driving mechanisms for this increased risk of CAD. Methods: We reviewed a Nationwide Inpatient Sample from 2007 to 2017, using Acute Coronary syndrome as a principal diagnosis with CD as the secondary diagnosis, utilizing validated ICD-9-CM and ICD-10 codes. We examined the annual trends in the number of cases and hospitalization charges yearly and used survey regression to calculate adjusted odds ratios (aOR) for hospital mortality and other outcomes. Results: We identified a total of 8,036,307 ACS hospitalizations from 2007 to 2017, of which 5917 (0.07\%) had a diagnosis for CD. The proportion of patients with CD in ACS hospitalizations increased from $0.015 \%$ in 2007 to $0.076 \%$ in 2017 . These patients were significantly older (70.3 vs. 67.4 years, $p<0.02)$, more likely female $(51.9 \%$ vs. $39.5 \%, p<0.01)$, and more likely to be white $(93.8 \%$ vs. $76.6 \% ; p<0.01)$ than ACS patients without CD. After adjusting for age, gender, race, Charlson Comorbidity index and hospital level characteristics, ACS hospitalizations for CD patients had a lower odds ratio for hospital mortality $(\mathrm{aOR}=0.39 ; 95 \% \mathrm{CI}=0.23-0.67 ; p<0.01)$. Additionally, length of stay in this patient population was shorter ( 4.53 vs. 4.84 days, $p<0.01$ ) but the mean hospitalization charges were higher (USD 64,058 vs. USD 60,223, $p<0.01$ ). Conclusion: We found that the number of ACS-related admissions in CD patients has risen more than five-fold between 2007 and 2017. However, the odds of in-hospital mortality in these patients is not higher than patients without CD. The results of our study demonstrate that although the systemic inflammation related to $\mathrm{CD}$ is associated with an increasing prevalence of ACS hospitalizations, on the contrary, the mortality rate is significantly higher in patients without celiac disease.
\end{abstract}

Keywords: celiac disease; myocardial infarction; database analysis; big data study; national inpatient sample

\section{Introduction}

While inflammation over a short period of time can serve as a benefit in mounting a defensive response and facilitating tissue repair, long-term inflammatory states have proven 
to be maladaptive [1]. Coronary artery disease (CAD) is thought to be typically associated with chronic pro-inflammatory states, as cytokines and interleukins are involved in multiple steps of atherogenesis, including endothelial cell dysfunction, plaque formation, plaque progression and rupture [2-4]. Celiac disease, a chronic autoimmune inflammatory disease, affecting the small intestine, is one such example of a maladaptive chronic inflammatory response [5]. The pathogenesis of coronary artery disease in celiac disease is not well identified. The increased cardiovascular risk is attributed to complex pathophysiological mechanisms that involve several signaling pathways with a contribution of various cell types and cytokines. Moreover, celiac disease is associated with a malabsorptive state, which may hinder absorption of cardioprotective nutrients and/or cardiac medications. For example, hyperhomocysteinemia, secondary to the malabsorption of folic acid and vitamin B12, might increase the risk of CAD.

It is postulated that these factors can cumulatively affect the risk of cardiovascular disease [6,7]. In this study, we aim to examine the incidence, trend and outcomes of Acute coronary syndrome (ACS) in patients with celiac disease.

\section{Methods}

We performed a retrospective, observational study using data from the Nationwide Inpatient Sample (NIS) (Healthcare Cost and Utilization Project, Agency for Healthcare Research and Quality), an administrative database consisting of (until 2012) all hospitalizations drawn from a sample of $20 \%$ of US hospitals, which are then weighted to be nationally representative of all US hospitalizations [5].

Our principal analysis included all adult hospitalizations with ACS as the primary discharge diagnosis using International Classification of Diseases, Ninth Revision; Clinical Modification (ICD-9-CM) codes (410.10X and 411.1) for years 2007-2014, ICD-10 codes for years 2016-2017 and a combination of ICD-9 and ICD-10 for 2015. The study cohort and ICD-9 codes used have been previously validated [5]. We then divided the ACS population into the two cohorts: one with baseline celiac disease, and another without celiac disease using ICD-9 (5790) or ICD-10 (K900) codes in any of the secondary discharge diagnoses.

\subsection{Study Variables and Outcomes}

NIS contains baseline patient characteristics such as age, sex, race/ethnicity, median yearly income in the patient's zip code, patient's comorbidities (Deyo adaptation of the Charlson Comorbidity Index for administrative data), hospital location (rural or urban), geographic region (Northeast, Midwest, West, or South), hospital teaching status, and hospital bed size. Length of hospital stay and total hospitalization charges were provided within the NIS for each hospitalization.

The primary outcome was in-hospital mortality, which was provided within the NIS for each discharge. Secondary outcomes were the length of hospital stay, total admission charges, discharge disposition and inpatient complications. These outcomes were compared between those with and without celiac disease who were admitted with ACS. We also trended the total number of hospitalizations of our individual study populations from 2007 to 2017.

\subsection{Statistical Analysis}

Multivariable regression analysis models were used to adjust the results for potential confounders. Multivariable regression models were built by including all confounders that were significantly associated with the outcome of univariable analysis using a cutoff $p$-value of 0.05 . Variables that were deemed important determinants of the outcomes based on literature review were incorporated into the models. Logistic regression as used for binary outcomes and linear regression was utilized for continuous outcomes. Proportions were compared by using the Fisher exact test, and continuous variables were compared by using the Student t-test. All $p$ values were two-sided, with 0.05 as the threshold for 
statistical significance. Patients with missing information for any of the variables in the regression analyses were excluded. Analyses were performed by using STATA version 15.0.

\section{Results}

\subsection{Population Demographics and Comorbidities}

Between January 2007 and December 2017, a total of 78 million patients were discharged from the studied US hospitals, $10.3 \%$ or $8,036,307$ patients were admitted with ACS and met our inclusion criteria. Of these, 5917 (0.07\%) had a secondary diagnosis of celiac disease. Table 1 presents a comparison of all hospitalizations with a primary diagnosis of ACS, regardless of a diagnosis of celiac disease. Patients with celiac disease presented with ACS at a significantly older age than those without (69.5 vs. 67.5 years respectively, $p<0.01)$. ACS patients with celiac disease were also more likely to be female $(52.7 \%$ vs. $39.4 \%, p<0.01)$. These patients also had a significantly higher prevalence of chronic obstructive pulmonary disease $(6.6 \%$ vs. $4.7 \%, p=0.021)$, alcoholism $(5.2 \%$ vs. $3.1 \%, p<0.01)$, family history of coronary artery disease $(12.4 \%$ vs. $10.3 \%, p=0.03)$, and systemic lupus erythematosus $(0.6 \%$ vs. $0.2 \%, p=0.04)$. ACS patients without celiac disease had a higher frequency of congestive heart failure (33.2\% vs. 29.4\%, $p=<0.0001)$, diabetes mellitus ( $37.3 \%$ vs. $28.8 \%, p<0.01)$, and tobacco smoking $(32.7 \%$ vs. $29.3 \%, p=0.027)$ than their counterparts with underlying CD. Finally, with regards to hospital characteristics, a greater number of celiac disease patients were treated at a teaching hospital (59.8\% vs. 56\%, $p=0.03)$.

\subsection{Trend of Hospitalizations}

Patients with celiac disease have shown a consistent rise in hospitalization rates, with a 3.07-fold increase from 2007 to 2017 (Figure 1). The increase in ACS hospitalizations was more pronounced in celiac disease patients than those without, an increase of 1.6-fold $(p=0.03)$. The increase in all-cause admissions for celiac disease patients was less than that for ACS, only increasing by 2.04-fold in the same 10-year period, between 2007 and 2017. The year with the most ACS admissions was 2017. Inpatient mortality rates have been consistent with the rate of hospitalizations over the study period, increasing by 4 .4-fold for the celiac disease cohort with ACS, and 1.84-fold for the non-celiac disease cohort.

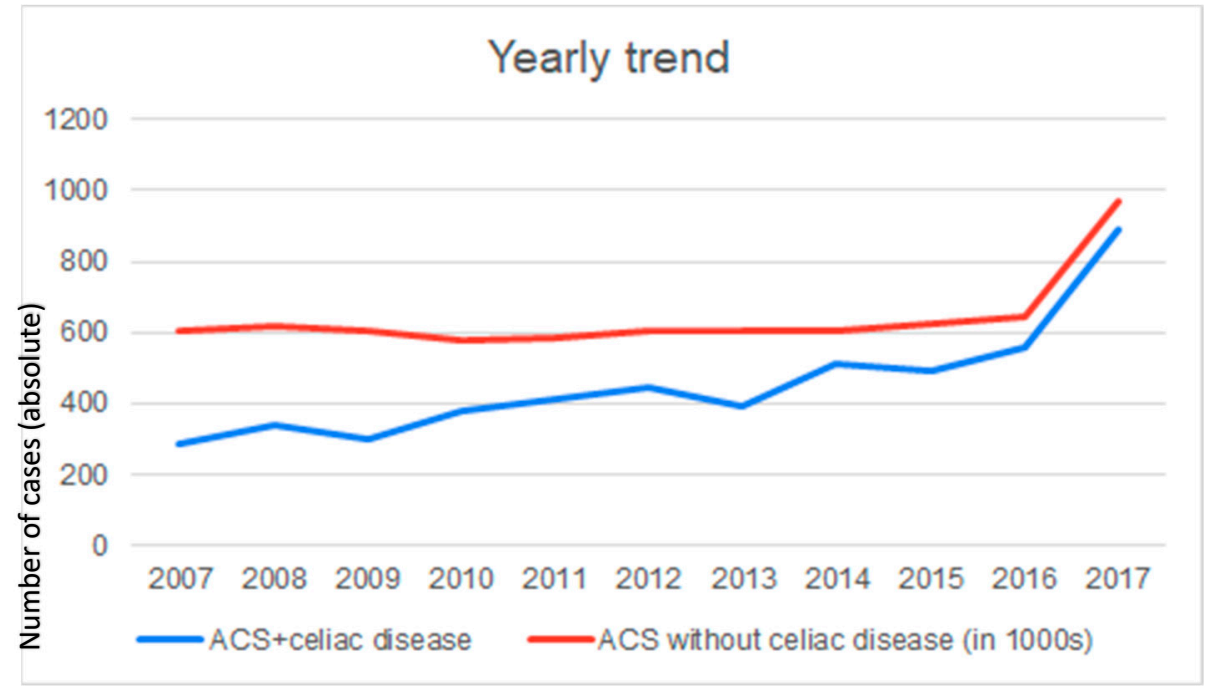

Figure 1. Yearly trend of ACS in celiac disease patients compared to non-celiac disease. (y axis is number of cases; in 1000 for ACS with no celiac disease). 
Table 1. Baseline characteristics of patients with ACS and with/without celiac disease.

\begin{tabular}{|c|c|c|c|}
\hline Variable & Patients with Celiac Disease & Patients without Celiac Disease & $p$ Value \\
\hline No. of patients & 5917 & $8,030,390$ & \\
\hline ACS $\%$ & 24.5 & 22.07 & \\
\hline \multicolumn{4}{|c|}{ Patient Characteristics } \\
\hline Female \% & 52.7 & 39.4 & $<0.001$ \\
\hline Age in years $(\mathrm{yr})$ & 69.5 & 67.5 & \\
\hline \multicolumn{4}{|l|}{ Age divided into categories $\%$} \\
\hline $18-35$ & 0.4 & 0.9 & 0.182 \\
\hline $36-45$ & 2.6 & 4.4 & 0.08 \\
\hline $46-65$ & 32.7 & 37.3 & $<0.001$ \\
\hline$>66$ & 64.2 & 57.3 & $<0.001$ \\
\hline \multicolumn{4}{|l|}{ Race \% } \\
\hline White & 78.6 & 72.3 & 0.008 \\
\hline Black & 0 & 8.6 & $<0.001$ \\
\hline Hispanic & 10.7 & 11.4 & 0.07 \\
\hline Other & 11 & 7.3 & $<0.001$ \\
\hline Charleston comorbidity index \% & & & 0.746 \\
\hline 0 & 2 & 1.9 & \\
\hline 1 or 2 & 53.7 & 52.5 & \\
\hline 3 or more & 44.2 & 45.4 & \\
\hline Median income in patient zip code $\%$ & & & $<0.001$ \\
\hline$\$ 1-\$ 38,999$ & 19.4 & 29.9 & \\
\hline$\$ 39,000-\$ 47,999$ & 25.7 & 27.4 & \\
\hline$\$ 48,000-\$ 62,999$ & 29.1 & 23.4 & \\
\hline$>\$ 63,000$ & 25.7 & 19.1 & \\
\hline Insurance Provider \% & & & $<0.001$ \\
\hline Medicare & 67.2 & 60.2 & \\
\hline Medicaid & 5.8 & 7.7 & \\
\hline Private & 25.4 & 26.5 & \\
\hline Uninsured & 1.4 & 5.5 & \\
\hline \multicolumn{4}{|c|}{ Hospital Characteristics \% } \\
\hline Teaching hospital & 59.8 & 56 & 0.032 \\
\hline Hospital size & & & 0.602 \\
\hline Small & 12.2 & 13.1 & \\
\hline Medium & 28.2 & 26.8 & \\
\hline Large & 59.5 & 60 & \\
\hline \multicolumn{4}{|c|}{ Patient Comorbidities \% } \\
\hline Congestive heart failure & 29.4 & 33.2 & $<0.001$ \\
\hline End stage renal disease & 11.9 & 14 & 0.056 \\
\hline Chronic obstructive pulmonary disease & 6.6 & 4.7 & 0.021 \\
\hline Coronary artery disease & 28.5 & 28.1 & 0.835 \\
\hline Obstructive sleep apnea & 3.4 & 2.9 & 0.39 \\
\hline Diabetes & 28.8 & 37.3 & $<0.001$ \\
\hline Hypertension & 45.5 & 47.7 & 0.182 \\
\hline Hyperlipidemia & 49.4 & 52.5 & 0.068 \\
\hline Atrial Fibrillation & 20.4 & 18.7 & 0.187 \\
\hline Aortic stenosis & 3.5 & 4 & 0.414 \\
\hline Alcohol & 5.2 & 3.1 & 0.001 \\
\hline Peripheral vascular disease & 8.3 & 6.7 & 0.058 \\
\hline Anemia & 15.9 & 10.4 & $<0.001$ \\
\hline Smoking & 29.3 & 32.7 & 0.027 \\
\hline family history of CAD & 12.4 & 10.2 & 0.034 \\
\hline Rheumatoid Arthritis & 1 & 0.9 & 0.68 \\
\hline Systemic lupus erythematosus & 0.6 & 0.2 & 0.04 \\
\hline Hypothyroidism & 21.3 & 11 & $<0.001$ \\
\hline
\end{tabular}

\subsection{Outcomes}

As shown in Table 2, the all-cause mortality rate for patients admitted with ACS who had underlying celiac disease was $0.6 \%$, as opposed to $0.8 \%$ in non-celiac disease patients 
( $p=0.004)$. The average length of stay (LOS) was significantly lower in the celiac disease vs. the non-celiac disease group ( $5.1 \mathrm{~d}$ vs. $10.2 \mathrm{~d}, p=0.036$ ). Mean total hospitalization charges (USD 68,400 vs. USD 72,184, $p=0.27$ ) were lower for ACS admissions in celiac disease patients but not significantly different from patients without celiac disease. Similarly, these patients were less likely to be discharged to rehabilitation centers and skilled nursing facilities $(11.7 \%$ vs. $20 \%, p=0.185)$, though this difference also had no statistical significance. In-hospital complication rates were not different between ACS patients with and without celiac disease, namely hemodynamic shock ( $3.2 \%$ vs. $2.6 \%, p=0.271)$, prolonged mechanical ventilation $>72 \mathrm{~h}(0.6 \%$ vs. $1.2 \%, p=0.071)$ and requirement for blood product transfusion $(5.4 \%$ vs. $4.8 \%, p=0.356)$.

Table 2. Comparison of celiac disease and non-celiac disease patients.

\begin{tabular}{cccc}
\hline & Celiac Disease & Non Celiac Disease Patients & $p$ Values \\
\hline Total ACS cases & 5917 & $8,030,390$ & \\
Total inpatient mortality & $40(0.6)$ & $68,144(0.8)$ & $<0.004$ \\
Total Charge & $68,400 \pm 2873$ & $72,184 \pm 604$ & 0.27 \\
Mean LOS & $4.54 \pm 0.14$ & $4.84 \pm 0.01$ & 0.036 \\
Length of stay by category & & & \\
$<3$ days & $55.60 \%$ & $54.99 \%$ & 0.042 \\
3-5 days & $17.55 \%$ & $19.09 \%$ & 0.03 \\
$>5$ days & $26.81 \%$ & $25.92 \%$ & 0.08 \\
Rehab Transfer & 11.7 & 20 & 0.185 \\
Shock & Complications & & 0.271 \\
Prolonged Mechanical & 3.2 & 2.6 & 0.071 \\
Ventilation & 0.6 & 1.2 & 0.356 \\
Requiring blood transfusion & 5.4 & 4.8 &
\end{tabular}

In terms of the multivariable regression, increasing age (per one year of increase, OR 1.11 with $95 \%$ CI 1.03-1.19, $p=0.004$ ) and a higher mean Charlson comorbidity score (OR 1.40 with $95 \%$ CI 1.05-1.87, $p=0.019$ ) both had significantly higher odds of in-hospital mortality in ACS patients with underlying celiac disease.

\section{Discussion}

Among celiac disease patients, $\mathrm{CV}$ involvement represents a topic of growing interest. In this large nationally representative analysis of patients with ACS, only a small proportion of patients $(0.07 \%)$ had underlying celiac disease. While still rare, there has been a steady rise in the prevalence of ACS in celiac disease patients, with a 3-fold increase demonstrated over the study period. This correlates with the increasing incidence of celiac disease in the 21 st century, at a rate of approximately $8 \%$ every year [8].

We identified geographical and socioeconomic factors, in addition to hospital-related factors, to demonstrate differences between patients with and without celiac disease who present with ACS. Females constituted a little over half the celiac disease patients with ACS, as compared to $40 \%$ of the ACS group without celiac disease. Though females have double the incidence of celiac disease compared to males, it is in fact males who have a higher preponderance for coronary artery disease $[9,10]$. There was no difference in the mean Charlson comorbidity burden between the two groups, which may suggest that our findings are possibly directly related to the presence versus absence of underlying celiac disease. Of the comorbid conditions, anemia was more common in celiac disease patients, which is likely explained by iron and cyanocobalamin (vitamin B12) malabsorption [11]. Prior studies have demonstrated a link between elevated homocysteine levels and risk for ischemic vascular disease [12], which is seen in celiac disease patients with vitamin B12 and/or folate deficiency. Unsurprisingly, other autoimmune conditions, such as hypothyroidism and systemic lupus erythematosus, were both more prevalent in the celiac disease group, confirming European registry data [13,14]. An interesting finding, however, 
was the lower prevalence of type 2 diabetes mellitus (DM) in the celiac disease group, a finding that contradicts previous data out of Finland, showing an equal prevalence in celiac disease patients and the general population [15]. Additional classic cardiac risk factors, such as hypertension and hyperlipidemia, were equal between both groups, with age at time of ACS presentation being 2 years higher in the celiac disease population. The latter finding is intriguing, given the usual early onset of celiac disease with progressively increased ACS risk hypothesized with passage of time, due to ongoing inflammation. Additionally, the celiac disease population with ACS was noted to belong to a higher income quintile compared to the non-celiac disease population, and they were also more likely to be admitted to an academic hospital.

Previous literature has shown an increased risk of incident ischemic heart disease (IHD) and death resulting from IHD in celiac disease patients [16-18]. This association seems to be primarily linked to systemic inflammation, with widespread immune activation playing a pivotal role in the process $[19,20]$. We demonstrated lower inpatient mortality in patients with celiac disease presenting with ACS, almost a third lower than patients without celiac disease. Length of stay was also shorter for ACS patients with celiac disease than for those without. It is important to note, however, that an administrative database, such as the NIS, is incapable of gauging disease severity and/or the complexities of an individual patient's hospital course [21], both of which are important determinants of mortality risk and length of stay.

Our study has several strengths and multiple limitations. It is a large nationwide analysis, taking into account patient characteristics and outcomes within different hospital systems and geographic regions. It is the first study of its kind, at this magnitude, to examine the correlation between the presence of celiac disease and patient outcomes in the setting of ACS. Inherent to the nature of our retrospective discharge database however, our analysis is limited by coding errors and missing data. Furthermore, we were unable to identify interventions performed to treat ACS, which largely influences outcomes. Although the sensitivity (94\%) and specificity ( $99 \%$ ) of ICD-9 codes for the identification of patients with a discharge diagnosis of ACS is established, those parameters have not been examined within the population of celiac disease patients with ACS [20]. Regardless, our study highlights important parameters related to the characteristics and outcomes of celiac disease patients hospitalized with ACS.

\section{Conclusions}

The ACS-related admissions of CD patients has risen more than five-fold between 2007 and 2017. However, the odds of in-hospital mortality in these patients is not higher than patients without $\mathrm{CD}$. The results of our study demonstrate that although the systemic inflammation related to $\mathrm{CD}$ is associated with an increasing prevalence of ACS hospitalizations, the mortality rate is significantly higher in patients without celiac disease. The clinical relevance of the observed differences may be minimal and should serve as a reassurance of comparable risk between patients with and without $C D$.

Author Contributions: K.S. and K.G. contributed equally to this manuscript and should be considered co-first authors. K.G. and S.P. devised the statistical analysis plan, wrote the statistical code and contributed in writing the manuscript. K.S. conceived the study idea and contributed in writing the manuscript. K.S. ran the statistical tests. M.A. contributed in review of the literature and writing the manuscript. M.A.-A. and K.N. contributed in review of the literature and writing the manuscript. A.K. was our faculty mentor who revised and edited the final manuscript. All authors provided critical feedback and helped shape the research analysis and manuscript. All authors have read and agreed to the published version of the manuscript.

Funding: The authors declare that there was no grant or financial support received whatsoever for the research conducted or the writing of this article.

Conflicts of Interest: All the listed authors declare that they have no conflict of interest. 
Statement of Ethics: This paper is exempt from ethical committee approval as it does not contain any studies involving human participants or animals. The database used for the study only includes de-identified data.

\section{References}

1. Willerson, J.T.; Ridker, P.M. Inflammation as a Cardiovascular Risk Factor. Circulation 2004, 109, II-2-II-10. [CrossRef]

2. Libby, P. Inflammation and cardiovascular disease mechanisms. Am. J. Clin. Nutr. 2006, 83, 456S-460S. [CrossRef]

3. Black, P.H.; Garbutt, L.D. Stress, inflammation and cardiovascular disease. J. Psychosom. Res. 2002, 52, 1-23. [CrossRef]

4. Liu, J.; Wang, H.; Li, J. Inflammation and Inflammatory Cells in Myocardial Infarction and Reperfusion Injury: A Double-Edged Sword. Clin. Med. Insights Cardiol. 2016, 10, CMC-S33164. [CrossRef]

5. Ludvigsson, J.F.; James, S.; Askling, J.; Stenestrand, U.; Ingelsson, E. Nationwide cohort study of risk of ischemic heart disease in patients with celiac disease. Circulation 2011, 123, 483-490. [CrossRef]

6. Ciaccio, E.J.; Lewis, S.K.; Biviano, A.B.; Iyer, V.; Garan, H.; Green, P.H. Cardiovascular involvement in celiac disease. World J. Cardiol. 2017, 9, 652-666. [CrossRef]

7. Mormile, R. Celiac Disease and Ischemic Heart Disease: What is the Link? J. Clin. Cell. Immunol. 2013, 4, 1-2. [CrossRef]

8. King, J.A.; Jeong, J.; Underwood, F.E.; Quan, J.; Panaccione, N.; Windsor, J.W.; Coward, S.; Debruyn, J.; Ronksley, P.E.; Shaheen, A.-A.; et al. Incidence of Celiac Disease Is Increasing Over Time: A Systematic Review and Meta-analysis. Am. J. Gastroenterol. 2020, 115, 507-525. [CrossRef]

9. Aggarwal, A.; Srivastava, S.; Velmurugan, M. Newer perspectives of coronary artery disease in young. World J. Cardiol. 2016, 8, 728-734. [CrossRef]

10. Bledsoe, A.C.; King, K.S.; Larson, J.J.; Snyder, M.; Absah, I.; Choung, R.S.; Murray, J.A. Micronutrient Deficiencies Are Common in Contemporary Celiac Disease Despite Lack of Overt Malabsorption Symptoms. Mayo Clin. Proc. 2019, 94, 1253-1260. [CrossRef]

11. Lerner, A.; Miri, B. Hypercoaglulability in celiac disease-an update. Autoimmun. Rev. 2014, 13, 1138-1141. [CrossRef]

12. Ludvigsson, J.F.; Rubio-Tapia, A.; Chowdhary, V.; Murray, J.A.; Simard, J.F. Increased Risk of Systemic Lupus Erythematosus in 29,000 Patients with Biopsy-verified Celiac Disease. J. Rheumatol. 2012, 39, 1964-1970. [CrossRef]

13. Ch'Ng, C.L.; Jones, M.K.; Kingham, J.G.C. Celiac Disease and Autoimmune Thyroid Disease. Clin. Med. Res. 2007, 5, 184-192. [CrossRef]

14. Kylökäs, A.; Kaukinen, K.; Huhtala, H.; Collin, P.; Mäki, M.; Kurppa, K. Type 1 and type 2 diabetes in celiac disease: Prevalence and effect on clinical and histological presentation. BMC Gastroenterol. 2016, 16, 76. [CrossRef]

15. Viljamaa, M.; Kaukinen, K.; Pukkala, E.; Hervonen, K.; Reunala, T.; Collin, P. Malignancies and mortality in patients with coeliac disease and dermatitis herpetiformis: 30-year population-based study. Dig. Liver Dis. 2006, 38, 374-380. [CrossRef]

16. Whorwell, P.; Alderson, M.; Foster, K.; Wright, R. Death from ischæmic heart-disease and malignancy in adult patients with cœliac disease. Lancet 1976, 308, 113-114. [CrossRef]

17. Gajulapalli, R.D.; Pattanshetty, D.J. Risk of coronary artery disease in celiac disease population. Saudi J. Gastroenterol. 2017, 23, 253-258. [CrossRef]

18. Korkmaza, H.; Sozenb, M.; Kebapcilar, L. Increased arterial stiffness and its relationship with inflammation, insulin, and insulin resistance in celiac disease. Eur. J. Gastroenterol. Hepatol. 2015, 27, 1193-1199. [CrossRef]

19. Rybak, A.; Cukrowska, B.; Socha, J.; Socha, P. Long Term Follow Up of Celiac Disease—Is Atherosclerosis a Problem? Nutrients 2014, 6, 2718-2729. [CrossRef]

20. Li, M.; Li, S.; Du, X.; Wu, T.; Li, X.; Ma, C.; Huo, Y.; Hu, D.; Gao, R.; Wu, Y. Factors attributed to the higher in-hospital mortality of ST elevation myocardial infarction patients admitted during off-hour in comparison with those during regular hour. PLoS ONE 2017, 12, e0175485. [CrossRef]

21. Metcalfe, A.; Neudam, A.; Forde, S.; Liu, M.; Drosler, S.; Quan, H.; Jetté, N. Case Definitions for Acute Myocardial Infarction in Administrative Databases and Their Impact on In-Hospital Mortality Rates. Health Serv. Res. 2013, 48, 290-318. [CrossRef] 